\title{
Local Search for Ant Colony System to Improve the Efficiency of Small Meander Line RFID Antennas
}

\author{
Gerhard Weis, Andrew Lewis, Marcus Randall, Amir Galehdar and David Thiel
}

\begin{abstract}
The efficient design of meander line antennas for RFID devices is a significant real-world problem. Traditional manual tuning of antenna designs is becoming impractical for larger problems. Thus the use of automated techniques, in the form of combinatorial search algorithms, is a necessity. Ant colony system (ACS) is a very efficient meta-heuristic that is commonly used to solve path construction problems. Apart from its own native search capacity, ACS can be dramatically improved by combining it with local search strategies. As shown in this paper, applying local search as a form of structure refinement to RFID meander line antennas delivers effective antenna structures. In particular, we use the operator known as backbite, that has had previous application in the construction of self-avoiding walks and compact polymer chains. Moreover, we apply it in a novel, hierarchical manner that allows for good sampling of the local search space. Its use represents a significant improvement on results obtained previously.
\end{abstract}

Keywords: ant colony system optimisation, backbite operator, RFID antenna design.

\section{INTRODUCTION}

$\mathbf{T}$ HE automated design of meander line RFID (radio frequency identification) antennas is relatively new. Traditionally, design engineers would create these manually in a time consuming iterative process. However, this is only practicable for small and relatively impractical antennas. Recent work has shown that heuristic search techniques are capable of producing very good antenna designs for larger problems [12]. This paper extends that work by considering a hierarchically structured local search refinement strategy. It is shown to be able to produce enhanced structures.

The idea of RFID was first developed in 1948 [15]. Recently there have been many enhancements to this basic idea and the concept has found applications in many areas. One suggestion is the replacement of the universal price code (UPC) barcode system. Practical considerations have driven a need for smaller tags with longer reading range. The read range can be defined as "the maximum distance at which an RFID reader can detect the backscattered signal from the tag" [13]. This vital factor can be increased by designing antennas with higher gain which is directly related to the antenna efficiency. Design engineers seek the smallest, most efficient antenna structures for their RFID tags.

Gerhard Weis and Andrew Lewis are with the Institute for Integrated and Intelligent Systems, Griffith University, Queensland, Australia (email: \{gerhard.weis@student., a.lewis@\}griffith.edu.au). Marcus Randall is with the School of Information Technology, Bond University, Queensland, Australia (email: mrandall@ bond.edu.au). Amir Galehdar and David Thiel are with the Centre for Wireless Monitoring and Applications, Griffith University, Queensland, Australia (email: \{s2145033@student., d.thiel@\}griffith.edu.au).
As mentioned above, one of the key features of RFID tag is antenna design. Traditionally, design engineers predict the performance of the antenna based on electromagnetic calculations which approximate real antenna performance. More recently, computers and accurate numerical modelling software applications have become available to explore antenna performance before construction. These codes are improved using mathematical optimisation techniques to produce the best possible solution. Many different optimisation techniques for antenna design are reported in the Special Issue of IEEE Transactions on Antennas and Propagation vol. 55 (3), March 2007. Meander line antennas are a subset of particular interest. An RFID system consists of two components:

- A transponder or tag - this has a small microchip and an antenna.

- A reader or scanner - this communicates with the tag and a host system which has the information of the identified tag item.

RFID tags are categorised into active and passive tags. Active tags have their own power source and passive tags harvest their's from the reader signal. Usually active and passive tags use different operating frequencies. Passive tags operating up to $100 \mathrm{MHz}$ work in the near field of the reader which means the range can be extended using lower frequencies. Since it is a near field operation, there are limits to the reading range.

Higher frequency tags operate beyond the near field of the reader, hence a longer reading can be achieved. Such tags use radio frequency backscatter which means the tag changes the antenna impedance by reflecting back some of the incident RF energy to the reader.

The antenna is the most crucial element for an RFID tag, because it is the power source, which makes good antenna gain essential in achieving a large transceiver range [7]. Other essential properties for an RFID antenna are size, robustness and manufacturing costs. The read range is directly proportional to the wavelength used. Lower frequencies or longer wavelengths tend to increase the read range, but also need larger antennas. The design goals for an RFID antenna is to make it small, efficient and with long reading distance.

Meander line antennas [18] are folded dipole antennas which increase radiation resistance without decreasing antenna bandwidth. They offer good radiation efficiency with considerable size reduction compared to conventional half or quarter wavelength antennas. Meander line antennas are of particular interest for RFID, due to their compactness and low manufacturing cost. 
There is no generally applicable analytic design methodology for meander line antennas. So there is a need for computational methods for design optimisation. There have been some approaches using genetic algorithms to optimise RFID antennas [8], [10], however, they have concentrated on simple serpentine configurations or on very specific application areas. To date there have been little or no investigations of general configurations or computational optimisation has not been applied [5], [6].

Ant colony system (ACS) is a constructive meta-heuristic search algorithm and is one of the family of ant colony optimisation (ACO) techniques. By its nature, it is ideally suited to solve path planning problems. Generating a meander line on a grid is a combinatorial problem that is a restricted and constrained form of the travelling salesman problem (TSP) hence it is $\mathcal{N P}$ hard [12]. There is a vast amount of literature covering ACO for the TSP (see Dorigo and Stützle [4] for an overiew). However, its use for antenna design has been limited to self structuring antennas [2].

In contrast to constructing meander line antennas, ACO for TSP attempts to find the shortest path to travel to all cities in a given set exactly once and return to the starting city. In TSP there is a connection between each pair of cities. Meander lines may only connect to a non-diagonal adjacent grid point within a bounded area. In other ways, producing meander lines is similar to constructing self avoiding walks (SAWs) [14]. A SAW is generally constructed on a grid of infinite size whereas meander line antennas are confined to a bounded area for which connections cannot wrap. Oberdorf, Ferguson, Jacobsen and Konedar [11] describe the compact polymer problem which closely resembles the construction of general meander lines on a grid. The main difference is that this work considers optimisation and refinement of the resultant structure, rather than the adequate sampling of the solution space of feasible compact polymers.

Randall, Lewis, Galehdar and Thiel [12] have successfully applied a grid-aware ACS algorithm to optimise meander line antennas. They examined the optimisation of antenna efficiency and constrained the solutions to being Hamiltonian walks. As the main goal of RFID antenna optimisation is to extend the read range of the RFID tag, it is essential to construct long antennas to keep the resonant frequency low. The longest path on a grid is a Hamiltonian walk. Their solution is very efficient for smaller grid sizes, but is less so for larger grid sizes. It is believed that local refinement of the structure may aid larger problems. Local search has already been successfully applied to ACO and the TSP (for example Stützle [16] and Stützle and Hoos [17]), where each ant can be guided by local refinement or a good solution created with ACO can be further optimised by local search. Mansfield [9] and Oberdorf et al. [11] describe an algorithm, called "backbite", to construct new Hamiltonian walks out of another Hamiltonian walk. As will be shown, this algorithm is directly applicable for local structure refinement in optimising meander antenna design.

The remainder of this paper is organised as follows. Sec- tion II describes the application of ACS to the design of meander lines while Section III shows how the backbite operator is applied and integrated into the system. The computational results of the new system are given and compared to those previously obtained by Randall et al. [12]. Finally, the conclusions and future research directions are presented in Section V.

\section{Ant Colony Systems for Meander Line ANTENNAS}

ACS is based on the capability of real ants to find the shortest path between a food source and their nest, without using visual cues. Instead, chemical markers known as pheromone are distributed throughout the environment to guide their paths. It has often been used by researchers and as such has proven good performance and robustness. As previously mentioned, it has been successfully used by Randall et al. [12] to optimise RFID meander line antennas.

ACS can best be described with the TSP metaphor as it is a well understood optimisation problem and, as previously shown, a close relation to the problem dealt with in this paper. Consider a TSP with $N$ cities. Cities $i$ and $j$ are separated by distance $d(i, j)$. Place $m$ ants randomly on these cities $(m \ll N)$. In discrete time steps, all ants select their next city then simultaneously move to their next city. Ants deposit an artificial pheromone to communicate with the colony about the utility (goodness) of the edges. A combination of the pheromone levels on the edges between the cities, the distance values of an edge is used to generate a set of probabilities from which the next city is chosen. Generally, the larger the pheromone value of an edge and the shorter the distance between the two cities, the higher the likelihood an ant will move along this edge. After all ants have visited all cities, global updating of the pheromone levels occurs. First, pheromone decays over time and second, edges that contribute to the best solution an ant has found are rewarded with an increase in pheromone level. A number of iterations are carried out until a given termination condition is satisfied. This could be, for instance, when a maximum number of iterations or a certain solution quality is reached. An in-depth description of the ACS algorithm for the TSP can be found in Dorigo and Gambardella [3].

The ACS algorithm needs to be adapted for the problem of creating meander line antennas [12]. Figure 1 (a) shows a $5 \times 5$ grid on which the ACS algorithm operates and (b) a possible solution. The ACS algorithm only solves one half of the antenna. The solution is then mirrored on the other half of the antenna, with a connecting $1 \mathrm{~mm}$ bridge. Each half area, or grid, contains $n \times n$ points. The physical size of the grid remains constant at $4 \mathrm{~mm}$, despite different values of $n$. As previously mentioned, each ant attempts to construct a meander line that begins on a boundary point and traverses all points on the grid.

The difficulty for the algorithm in constructing Hamiltonian walks (as reported by Randall et al. [12]) is that each point is only connected to at most four others. It is quite likely that an 


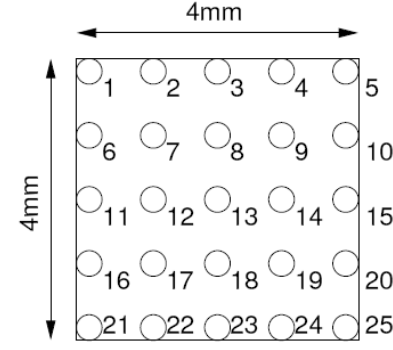

(a)

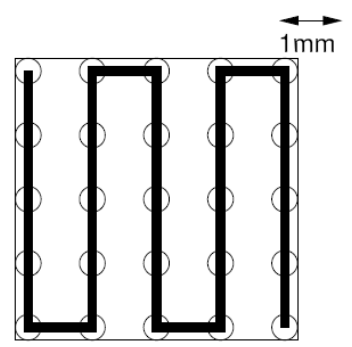

(b)
Fig. 1. (a) defines the grid and numbering system and (b) shows a possible meander line antenna. The latter is referred to as a "plough" structure.

ant will "trap" itself within the grid, as it has already visited all the neighbouring points.

To minimise the potential of an ant becoming stuck, the ACS algorithm for meander line antennas works in the following way. Each ant starts at a random chosen boundary point and walks to the next chosen neighbour inside the boundaries that are not already visited. The ants are guided by the following three factors:

1) Pheromone - The pheromone matrix is an $n^{2} \times 4$ structure. The latter dimension is direction. The pheromone component of the characteristic probablistic equations is given as $\tau(c, d)$ where $c$ is the current grid position and $d$ is the direction.

2) Lookahead function - In order to move each ant, the number of unused neighbours of each of the neighbours of the current point is calculated. The greater this number, the higher the probability of choosing that direction.

3) Straight line segment function - Encouraging straight line segments helps to ensure that all $n^{2}$ points are visited by the meander line. This is easily calculated by comparing the current candidate direction with the previous direction that the ant has taken.

If the number of unused neighbours for an ant is 0 , then the ant has become stuck and is considered dead (if not all points are visited by this ant). Dead ants are disregarded for the remainder of the iteration. At the end of each iteration, all ants that have valid solutions have these evaluated by the NEC [1] antenna suite. Therefore, the calculation of the efficiency of the generated antennas is a black box to this algorithm, and the ants can not use any incremental objective information as guidance.

\section{APPLYING THE BACKBITE OPERATOR}

Local search is an integral part of ACO, and on the whole, greatly improves solution quality [3], [16], [17]. As an example, Stützle and Hoos [16] applied local search for a $\mathcal{M} \mathcal{A} \mathcal{X}-\mathcal{M I N}$ Ant System to the TSP and the quadratic assignment problem (QAP). For both problems, local search improved the runtime and solution quality compared to an ant system without this local refinement.

Refinement of meander lines cannot use the standard local search operators that are typically applied to the benchmark problems such as TSP and QAP. However, a well known approach to generate new Hamiltonian walks from another Hamiltonian walk is the backbite operator [9], [14]. Figure 2 shows how the backbite operator works.

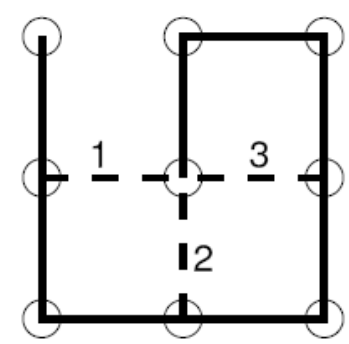

(a)

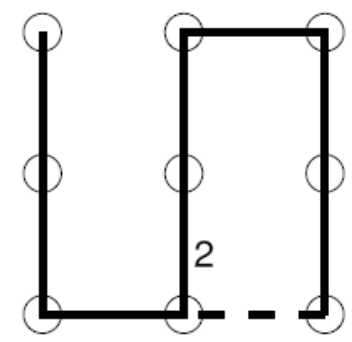

(c)

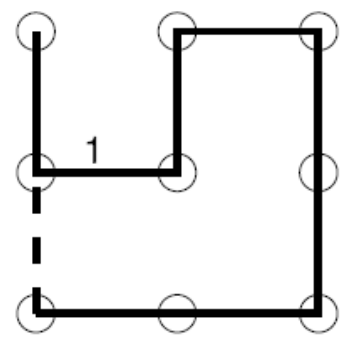

(b)

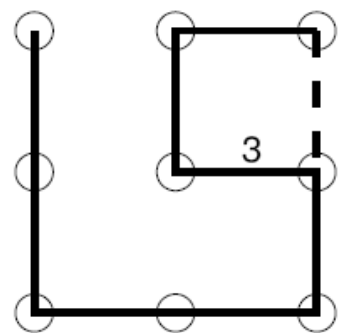

(d)
Fig. 2. (a) shows all possible backbite moves. (b) - (c) show all resulting structures after the backbite operation.

Figure 2 (a) is the starting structure with the starting point in the upper left corner and the end point in the centre. To construct a new Hamiltonian walk we look for unconnected neighbours at the end point. In this case there are three possibilities to insert a new connection. Adding a new connection will create a circuit, which means another connection has to be removed. In Figure 2 (b) connection 1 has been added and the dashed line has been removed. The new endpoint is in the lower left corner, where it is possible to do further backbite operations. (In Figure 2 (c) and 2 (d) connections 2 and 3, respectively, were inserted).

If further backbite operations are performed, they can be described and arranged as an $n$-ary tree, where $n$ has a maximum of 3 , deriving from one particular origin structure. Figure 3 shows a portion of such a tree. The nodes in the tree can be labelled using a notation indicating the possible inserted 


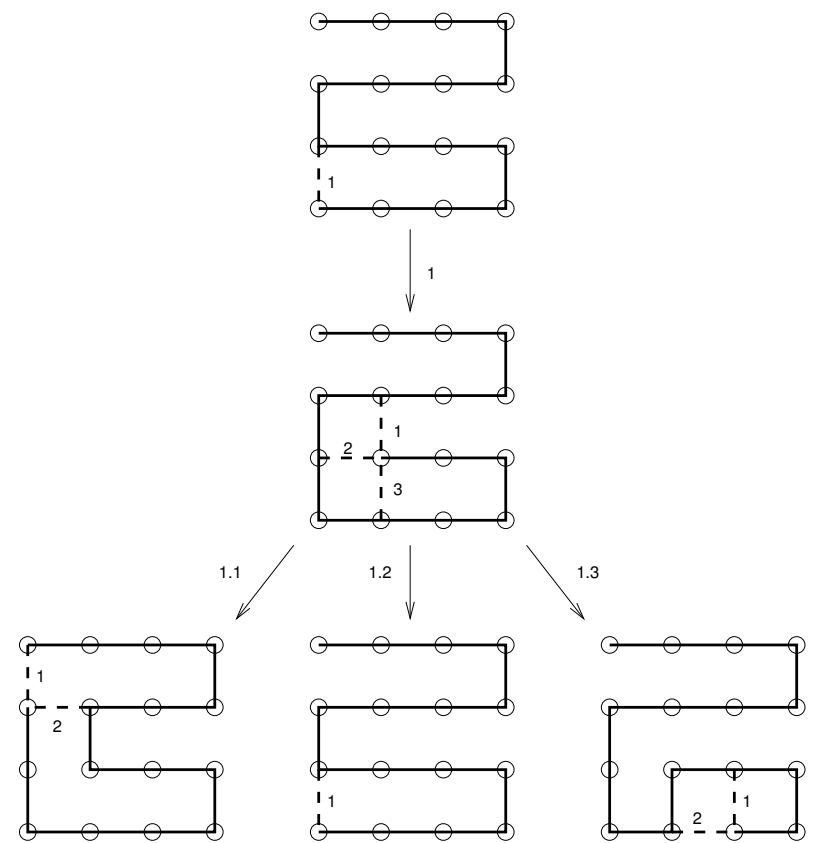

Fig. 3. The $n$-ary tree of possible structures derived using iterative backbite operations.

links at each stage. For example, a notation of 1.2 means that in the first step possibility 1 was used and in the following step possibility 2 was used. This tree, and labelling, can obviously be extended to arbitrary depth. In addition, it may be noted that all node structures can be fully and independently constructed based on the root node structure. A set of nodes to some predetermined depth can be constructed and all possibilities tested concurrently in a single evaluation step, given sufficient, available, parallel computing resources. It only remains to be careful to prune the tree of loops.

A single iteration of the ACS algorithm, including the backbite operator, is outlined in Algorithm 1. The procedure shown will be repeated for some specified, maximum number of iterations. As shown, the backbite operation was applied as a hierarchical search after the ACS algorithm has produced a final solution. The local search was restricted to a given hierarchy search depth. All resulting structures were generated and the efficiencies calculated for each structure concurrently. Since the performance of the refined structures are evaluated concurrently with the structure constructed by the ACS the additional evaluations required for refinement can be performed without adding to the time required for a single iteration of the algorithm, provided sufficient parallel computing resources are available.

\section{COMputational EXPERIMEnTS}

We solved for meander lines on grids ranging from $5 \times 5$ to $10 \times 10$, running the ant algorithm ten times (by varying the random initial seed used for the probabilistic operations of the algorithm). Each search was permitted 2000 iterations. Inspection of the results obtained showed that an iterative approach to application of the backbite operator - applying

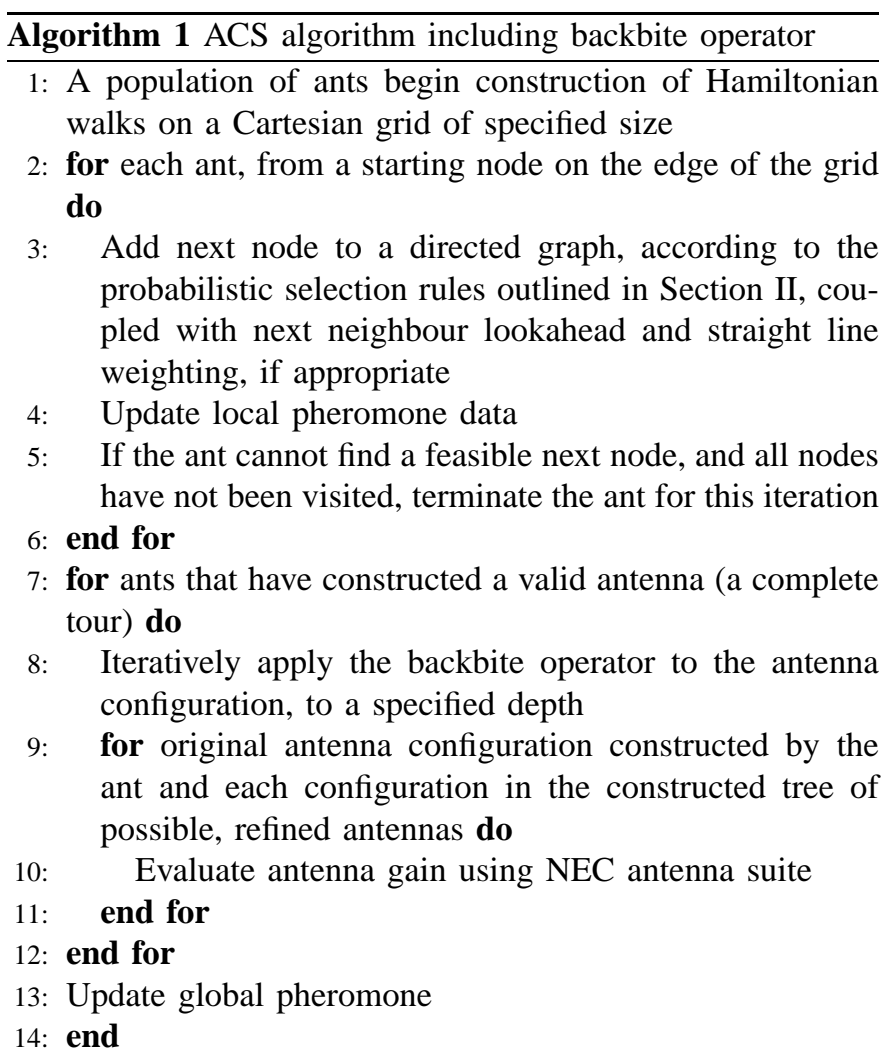

the operator, evaluating the resultant structures, and selecting the best for further refinement - was not, in fact, a desirable approach because efficiency can vary unpredictably between structures, leading to stagnation of the procedure in local optima. Construction of an entire tree of possible, refined configurations allowed the algorithm to search beyond intermediate structures of degraded performance.

For each grid size, the best antenna found was compared with those found without using the backbite operator. The following figures show the best structures found in previous work [12] on the left side and the structure further optimised using the backbite operator on the right side. The efficiency as a percentage is noted below each structure.

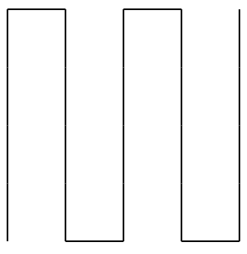

(a) 83.0

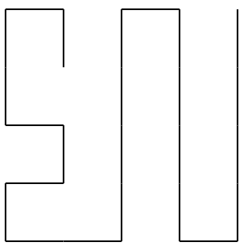

(b) 83.1
Fig. 4. (a) The original $5 \times 5$ antenna and (b) the optimised $5 \times 5$ antenna.

The $5 \times 5$ example in Figure 4 is quite impressive. Beginning with a search depth of 5 it took only 3 steps to find the global optimum as determined by Galehdar et al. [6] using exhaustive 
enumeration. The total number of antennas evaluated was 13 .

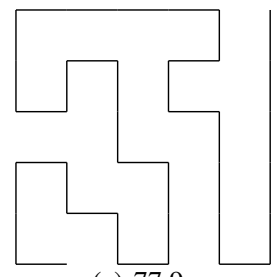

(a) 77.9

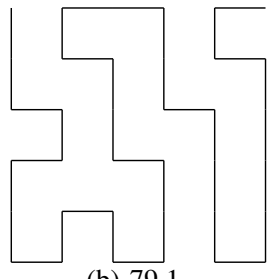

(b) 79.1
Fig. 5. (a) The original $6 \times 6$ antenna from "bendy" algorithm and (b) the optimised $6 \times 6$ antenna.

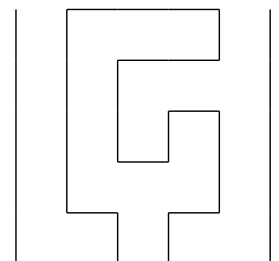

(a) 64.9

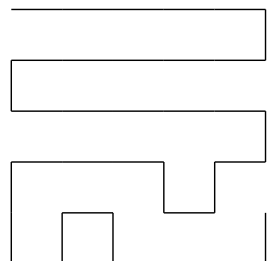

(b) 79.1
Fig. 6. (a) The original $6 \times 6$ antenna from the "straight" algorithm and (b) the optimised $6 \times 6$ antenna.

Figure 5 shows the results for the $6 \times 6$ antenna derived using the "bendy" algorithm described in earlier work [12] and Figure 6 shows the results for the $6 \times 6$ "straight line" antenna. For both the search depth was set to 25 . For the first antenna, the algorithm needed to evaluate 270 antennas and improved the antenna efficiency by $1.5 \%$. For the second structure, there were 395 antennas to evaluate. The efficiency was improved to $79.1 \%$, the same as for the structure in Figure 5 (b), a relative increase of $21.9 \%$. It might be inferred from this that these searches have both reached near-optimal results for this antenna size, but that the "bendy" algorithm achieved a better result originally.

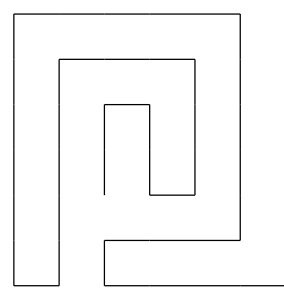

(a) 70.2

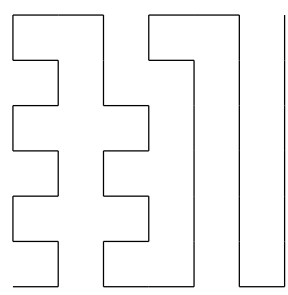

(b) 75.7
Fig. 7. (a) The original $7 \times 7$ antenna and (b) one of 7 equivalent, optimised $7 \times 7$ antennas.

For the $7 \times 7$ case the algorithm had 891 antennas to evaluate (for a search depth of 25) and found 7 different equally efficient antenna structures, one of which is shown in Figure 7. The increase in efficiency achieved was $7.8 \%$.
To reduce computation time the search depth for $8 \times 8$ grids was reduced to 20 . The local search had to evaluate 3193 different antennas and improved the efficiency $7.6 \%$. The evaluation of this, the largest group of structures, took about one and a half hours using 20 desktop computers (a mixture of Athlon 64 and Pentium 4 computers in an ad hoc cluster.)

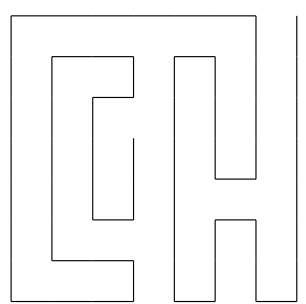

(a) 67.1

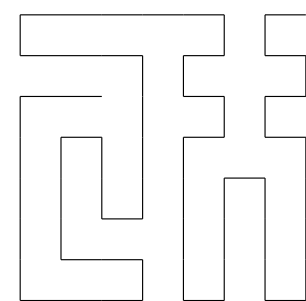

(b) 72.2
Fig. 8. (a) The original $8 \times 8$ antenna and (b) the optimised $8 \times 8$ antenna.

For the $9 \times 9$ grids, the search depth was further reduced, because larger grids take increasing compute time to evaluate. For a search depth of 15, 1371 antennas had to be evaluated. A solution was found with efficiency increased by $16.8 \%$ compared to the original structure.
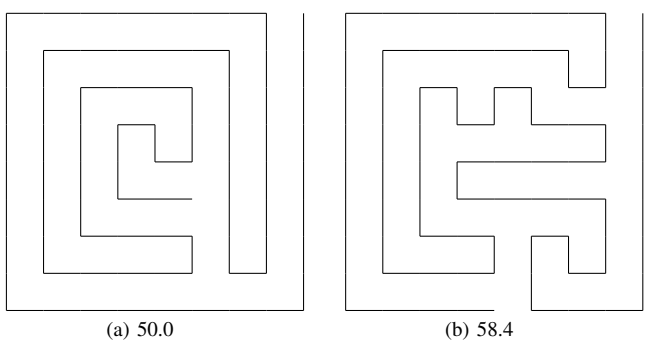

Fig. 9. (a) The original $9 \times 9$ antenna and (b) the optimised $9 \times 9$ antenna.

For $10 \times 10$ grid, the search depth was further reduced to 15 and 1527 new antennas were evaluated. The efficiency was improved notably by $42.7 \%$.
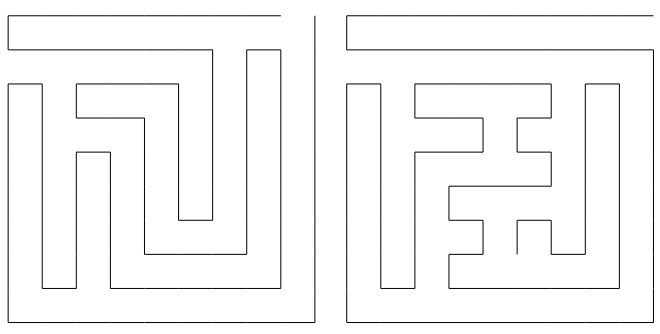

Fig. 10. (a) Original $10 \times 10$ antenna and (b) the optimised $10 \times 10$ antenna

In summary, use of the backbite operator for local structure refinement produced antennas with improved efficiency at all grid sizes. For smaller grids it either obtained the globally optimal solution, as verified by exhaustive enumeration, or can 
reasonably be assumed to have closely approached it for sizes for which complete enumeration of solutions is infeasible. It may be noted that in a number of cases, for these smaller grid sizes, the un-augmented algorithm had already derived very efficient structures. As grid sizes were increased, however, local refinement provided increasing degrees of improvement, indicating both the difficulty of the optimisation problem and the need for hybridisation with local search.

\section{Conclusions}

Overall it seems, that antennas with a serpentine part and another more "bendy" part are more efficient than the usual spiral and plough structures. The results show that the backbite operator applied in an hierarchical manner as a post-processing step is a suitable local search strategy for meander line antennas. Due to the independence of the evaluation function (i.e., the NEC tool), it is very easy to parallelise. Ideally, it would be preferable to be able to direct the local search more using domain specific knowledge, instead of generating a number of solutions and testing them. However, no relationship between a small change in structure and the resulting efficiency could be found within the testing performed. Generally, the test runs have shown that starting with a good structure and applying the backbite operator hierarchically leads to resultant structures of higher efficiencies.

Another issue of importance in this area is that of the resonant frequency of antenna operation. Future work will be directed to the development of multi-objective optimisation methods to simultaneously satisfy the criteria of efficiency and resonant frequency. Beyond this, we wish to investigate other constructive heuristics with which to build meander lines as a basis of comparison to ACO. It should be noted that to date few or no attempts have been made to apply computational optimisation methods to this problem.

\section{REFERENCES}

[1] G. Burke, A. Poggio, J. Logan, and J. Rockway. NEC - Numerical electromagnetics code for antennas and scattering. Antennas and Propagation Society International Symposium, 17:147-150, June 1979.

[2] C. Coleman, E. Rothwell, and J. Ross. Investigation of simulated annealing, ant-colony optimization, and genetic algorithms for selfstructuring antennas. Antennas and Propagation, IEEE Transactions on, 52(4):1007-1014, 2004.

[3] M. Dorigo and L. Gambardella. Ant Colony System: A cooperative learning approach to the traveling salesman problem. IEEE Transactions on Evolutionary Computation, 1(1):53-66, 1997.

[4] M. Dorigo and T. Stützle. Ant Colony Optimization. MIT Press, 2004.

[5] A. Galehdar, D. Thiel, and S. O'Keefe. Antenna efficiency calculations for electrically small, RFID antennas. IEEE Antenna and Wireless Propagation Letters, 6:156-159, 2007.

[6] A. Galehdar, D. Thiel, S. O'Keefe, and S. Lingsley. Efficiency variations in electrically small, meander line RFID antennas. In Proceedings of IEEE Antenna Propagation Symposium, 2007.

[7] M. Keskilammi, L. Sydänheimo, and M. Kivikoski. Radio frequency technology for automated manufacturing and logistics control. Part 1: Passive RFID systems and the effects of antenna parameters on operational distance. The International Journal of Advanced Manufacturing Technology, 21(10):769-774, 2003.

[8] G. Kim and Y. Chung. Optimization of UHF RFID tag antennas using a genetic algorithm. IEEE Antennas and Propagation Society International Symposium, pages 2087-2090, July 2006.

[9] M. Mansfield. Monte Carlo studies of polymer chain dimensions in the melt. The Journal of Chemical Physics, 77(3):1554-1559, 1982.
[10] G. Marrocco. Gain-optimized self-resonant meander line antennas for RFID applications. Antennas and Wireless Propagation Letters, 2:302305, 2003.

[11] R. Oberdorf, A. Ferguson, J. Jacobsen, and J. Kondev. Secondary structures in long compact polymers. Physical Review E (Statistical, Nonlinear, and Soft Matter Physics), 74(5):051801, 2006.

[12] M. Randall, A. Lewis, A. Galehdar, and D. Thiel. Using ant colony optimisation to improve the efficiency of small meander line RFID antennas. In $3^{\text {rd }}$ IEEE International e-Science and Grid Computing Conference, 2007.

[13] K. Seshagiri, R. Nikitin, and S. Lam. Antenna design for UHF RFID tags: A review and a practical application. IEEE Transactions on Antennas Propagation, 53:3870-3876, 2005.

[14] A. Sokal. Monte carlo methods for the self avoiding walk. Monte Carlo and Molecular Dynamics Simulations in Polymer Science, pages 47-124, March 1994.

[15] H. Stockman. Communication by means of reflected power. Proceedings of the Institute of Radio Engineers, pages 1196-1204, 1948.

[16] T. Stützle. The Max-Min Ant System and local search for combinatorial optimization problems. In S. Voss, S. Martello, I. Osman, and C. Roucairol, editors, Meta-heuristics: Advances and Trends in Local Search Paradigms for Optimization, pages 313-329. Kluwer, 1999.

[17] T. Stützle and H. Hoos. MAX-MIN Ant System and local search for the traveling salesman problem. In IEEE International Conference on Evolutionary Computation, pages 309-314. IEEE Press, April 1997.

[18] T. Warnagiris and T. Minardo. Performance of a meandered line as an electrically small transmitting antenna. Antennas and Propagation, IEEE Transactions on, 46(12):1797-1801, 1998. 